\title{
Reduced graphene oxide micropatterns as an interface for adherent cells
}

\author{
Lotta E. Delle ${ }^{1,2}$, Ruben Lanche ${ }^{1,2}$, Jessica Ka-Yan Law', Maryam Weil', Xuan Thang Vu', Patrick Wagner ${ }^{2}$, \\ and Sven Ingebrandt ${ }^{*, 1}$ \\ ${ }^{1}$ Department of Informatics and Microsystem Technology, University of Applied Sciences Kaiserslautern, Amerikastr. 1,
66482 Zweibrücken, Germany
${ }^{2}$ Institute for Materials Research, Hasselt University, Wetenschapspark 1, 3590 Diepenbeek, Belgium
}

Received 15 October 2012, revised 11 February 2013, accepted 11 February 2013

Published online 5 April 2013

Keywords cell adhesion, impedance spectroscopy, reduced graphene oxide, scanning electron microscopy

*Corresponding author: e-mail sven.ingebrandt@fh-kl.de, Phone: +49 3724 5413, Fax: +49 37245313

In the present study, reduced graphene oxide (rGO) is used as a biointerface for the investigation of cell adhesion of human embryonic kidney (HEK 293) cells. A fast, straightforward, and substrate-independent soft lithography approach known as "Micromolding In Capillaries" (MIMIC) was utilized to pattern graphene oxide (GO) arrays. Large-scale GO patterns with widths and distances in the micrometer range were obtained and were subsequently reduced to rGO via an environmentally-friendly procedure using L-ascorbic acid. Physical characterization of rGO patterns and cells was performed by optical microscopy, atomic force microscopy, and scanning electron microscopy. Impedance spectroscopy was used for the electrochemical characterization of GO before and after reduction. Cell adhesion and alignment was strong on the rGO micropatterns. In future assays, the rGO could combine two functions: cellular patterning and electrical interfacing of cells.

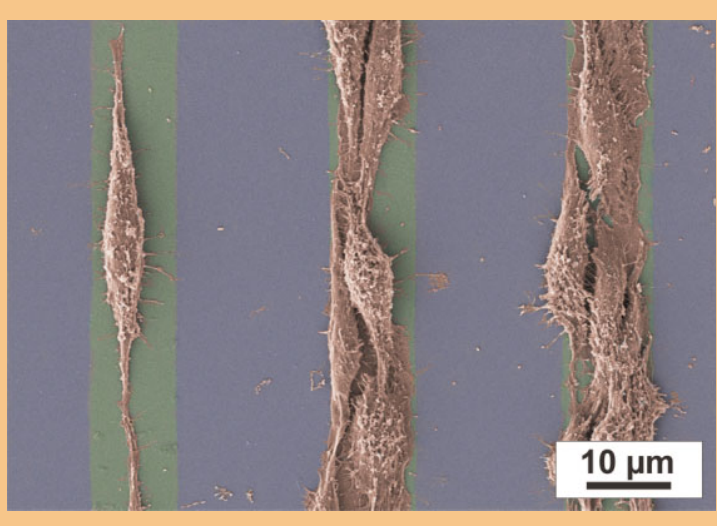

HEK 293 cells aligning on rGO micro patterns (SEM image)
1 Introduction Carbon-based nanomaterials such as nanocrystalline diamond (NCD) [1-3], carbon nanotubes (CNT), and graphene $[4,5]$ are known as biocompatible materials. The interface of graphene with living cells is regarded as a possible future generation of neuro-prosthetic devices [5], as it promises a further improvement of signal detection capability, stability in biological environments, and an improved biocompatibility with living tissue [6]. Therefore, the deep understanding of the graphene-cell interfaces is necessary in order to use graphene as an electronic transducer.
Coupling biological elements with graphene-based microelectronic devices using the electrical field effect [7], impedimetric [8], or amperometric [9] detection mechanisms is leading towards the formation of sensor interfaces with high sensitivity and selectivity. In combination with advanced signal amplification methods and processing, future systems might allow investigation of networks in neuron cultures and signal propagation inside tissue.

Graphene-based devices can be fabricated by mechanical exfoliation [10], thermal growth methods like chemical vapor deposition [11], or epitaxial growth of graphene [12]. 
All these methods target for defect-free, single-layer graphene sheets, which exhibit extremely high carrier mobility. An alternative fabrication route is the synthesis of graphene oxide (GO) by the modified hummers method using natural graphite and subsequent reduction into reduced graphene oxide (rGO), which is conductive as well. Although much lower carrier mobility can be obtained in these multi-layered material systems, the fabrication of rGO with thermal or chemical routes is cost-effective, massively scalable, and versatile for chemical functionalization [13]. Established methods to effectively reduce GO are the treatment with hydrazine or hydrazine hydrate [14]. Due to the fact that hydrazine is highly poisonous and explosive, special precautions have to be taken. Therefore, new approaches for the environmentally-friendly reduction of GO were investigated like the reduction with $\mathrm{L}$-ascorbic acid (L-aa) $[13,15,16]$. However, it is still a challenge to produce rGO in patterned structures. This is due to the fact that graphene is a hydrophobic material (and so is rGO, if an efficient reduction process was chosen) and solubility in aqueous liquids is poor. GO in turn is hydrophilic and can be easily solubilized in water.

The usage of GO in combination with a soft lithographic approach, called "Micromolding In Capillaries" (MIMIC) $[17,18]$, promises a straightforward and low-cost procedure for patterning large-scale GO lines. In the present study, patterned lines of GO with micrometer widths were produced via this MIMIC technique. We used an environmentally-friendly chemical reduction route using a solution of L-aa. The thus fabricated $\mathrm{rGO}$ patterns were highly uniform and stable. Characterization of the rGO pattern was performed using optical microscopy, atomic force microscopy (AFM), and scanning electron microscopy (SEM). The degree of GO reduction was followed by the changes in electrochemical impedance. In order to study the biocompatibility of the rGO patterns, HEK 293 cells were grown on top of the rGO. The morphology of cells on the rGO patterns was assessed by optical microscopy and SEM.

\section{Materials and methods}

2.1 Micropatterning of GO lines GO flakes (graphene-supermarket.com, USA) were dispersed in de-ionized (DI) water in a concentration of $2 \mathrm{mg} \mathrm{ml}^{-1}$ and agitated by ultrasonication (ELMA Transsonic digital, T $680 \mathrm{DH}$, $40 \mathrm{kHz}, 150$ Watt, USA) for $4 \mathrm{~h}$. Afterwards, sedimentation was allowed overnight and finally the upper part of the solution was collected and used for MIMIC.

Figure 1 shows an illustration of the MIMIC patterning of GO on glass substrates. The silicon master with line patterns of $2-50 \mu \mathrm{m}$ widths and $5 \mu \mathrm{m}$ height was fabricated by standard optical lithography followed by reactive ion etching. Polydimethylsiloxane (PDMS) (Sylgard 184, A:B 1:10, Dow Corning) stamps were fabricated using the negative silicon master $(2-50 \mu \mathrm{m}$ widths of patterns; Fig. 1A).

Prior to GO patterning, the PDMS stamp was cut at both ends to open the channels and the structured side of the stamp
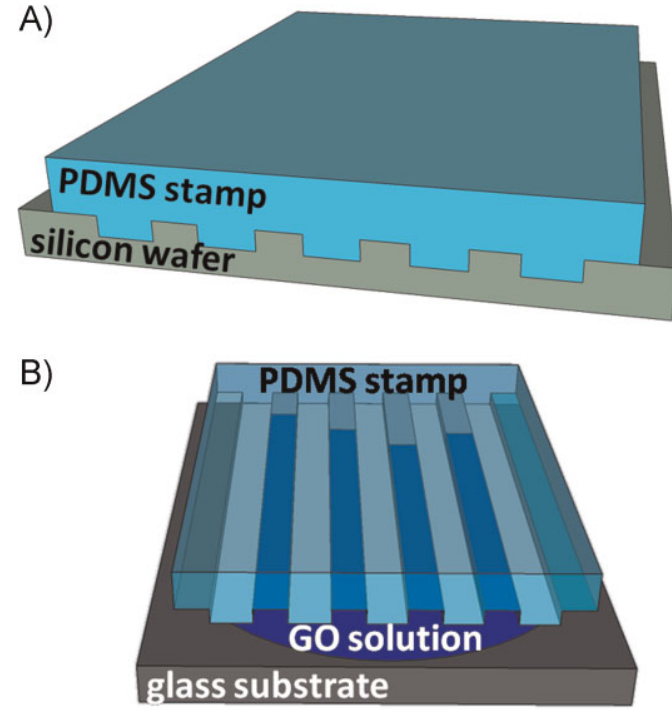

C)

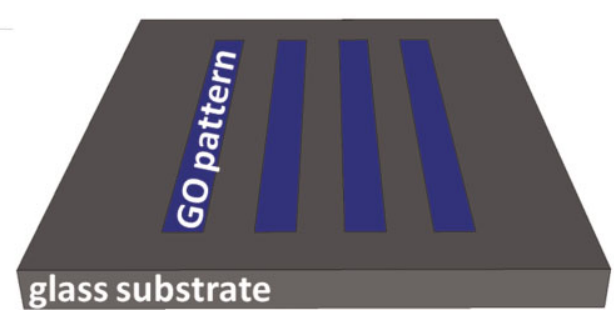

Figure 1 Illustration of the MIMIC technique for patterning GO lines. (A) Production of PDMS stamp on silicon master having a patterned relief structure. (B) Aligning the PDMS stamp on a glass substrate and applying a drop of GO solution at the open end of the channels. (C) After drying and removing the stamp, the GO patterns remain on the substrate.

was activated in $\mathrm{O}_{2}$ plasma ( $1 \mathrm{~min}, 0.6 \mathrm{mbar}, 140$ Watt) (Diener electronic $\mathrm{GmbH}+\mathrm{Co}$. KG, Germany) to hydrophilize the surface. As substrates we used either glass samples $\left(\right.$ Pyrex ${ }^{\circledR}$, Borofloat 33 borosilicate glass wafers, Silicon Materials Si-Mat, Germany) or oxidized silicon wafers $\left(300 \mathrm{~nm} \mathrm{SiO}_{2}\right.$ by wet thermal oxidation). The substrates were silanized with 3-aminopropyl-triethoxysilane (APTES, Sigma-Aldrich) in gas phase for $1.5 \mathrm{~h}$ at $150^{\circ} \mathrm{C}$ [19]. Afterwards, the PDMS stamp was placed onto the APTES-modified glass surface in order to form a conformal contact. The MIMIC method was performed by placing a drop of GO solution at the open ends of the channel. The channels were filled by capillary force (Fig. 1B). Degassing in a desiccator for 20 min supported the filling of the channels and removed enclosed air bubbles between stamp and substrate. After drying, the stamp was removed and patterned GO lines remained on the substrate (Fig. 1C).

2.2 Reduction to rGO The on-chip reduction of GO was performed by a solution of L-aa (Riedel de Haen, Germany) with a concentration of $10 \mathrm{mg} \mathrm{ml}^{-1}$ at room temperature. The patterned chip was stored in $4 \mathrm{ml}$ of the $\mathrm{L}$-aa 
solution for 1-7 days. The reduction was occurring due to the removal of oxygen functionalities in the GO. After the storage in L-aa solution, the chip was dried with nitrogen gas and left in an oven for $30 \mathrm{~min}$ at $100{ }^{\circ} \mathrm{C}$ to remove residual water.

2.3 Physical and electrical characterization All surfaces were characterized in terms of water contact angle using the sessile drop method (Dataphysics Instruments $\mathrm{GmbH}$, OCA 15 pro). Both GO and rGO patterns on glass substrates were characterized by optical microscopy using differential interference contrast (DIC), AFM (Dimension 3100, Digital Instruments, Germany) in tapping mode, and SEM (Zeiss Supra 40, Carl Zeiss AG, Germany). For electrical characterization, the GO lines at both ends of the patterns were commonly contacted using gold contacts ( $300 \mathrm{~nm}$ thickness) evaporated through a shadow mask. The reduction efficiency was monitored by impedance spectroscopy (IS) (Ivium CompactStat, Ivium Technologies BV, Netherlands) in a two electrode configuration.

2.4 Cell culture HEK 293 cells were cultured and maintained in an incubator at $37{ }^{\circ} \mathrm{C}$ with $5 \% \mathrm{CO}_{2}$. For standard culturing conditions, M10 medium (PAN, Germany) was used, containing $1 \%$ glutamine, $1 \%$ penicillin/ streptomycin (PAN, Germany), 10\% fetal calf serum (PAN, Germany), and $0.8 \mathrm{mg} \mathrm{ml}^{-1} \mathrm{G} 418$ (Carl Roth, Germany). HEK 293 cells were cultured on GO- and rGO-patterned glass chips. All experiments for the cell culture were performed on MIMIC samples with structures of $10 \mu \mathrm{m}$ width and $20 \mu \mathrm{m}$ spacing due to a higher reliability of these structures during MIMIC fabrication. The chips were sterilized under UV light for $30 \mathrm{~min}$ and $200 \mu \mathrm{l}$ of cell suspension (90,000 cells for SEM imaging and 50,000 cells for cell growth studies) were added on top of each glass chip. The chips were covered with $2 \mathrm{ml} \mathrm{M} 10$ medium after $4 \mathrm{~h}$. The medium was changed every 2 days during cell culture.

2.5 Cell growth quantification Microscopic images were taken every day from a defined area $(2.5 \mathrm{~mm} \times 3 \mathrm{~mm})$ on the same position of the samples up to 4 days in culture. Cells growing on top of the rGO patterns were counted in comparison to cells growing in between the patterns. Apart from the rGO patterning on the aminosilanized glass or $\mathrm{SiO}_{2}$ surfaces, no other cell adhesion promoting coatings were used in this study.

2.6 Fixation of cells for SEM images The fixation of the cells on the samples for the SEM imaging was done with glutaraldehyde (Sigma-Aldrich, Germany) [20] as a crosslinking agent. The fixation is based on a progressive dehydration of the cells in an ascending 2-propanol (SigmaAldrich, Germany) concentration in DI water.

After the removal of the medium, the chips with the cultured HEK 293 cells were rinsed twice with $500 \mu \mathrm{l}$ phosphate buffered saline (PBS). The chips were then immersed in a solution of $3 \%$ glutaraldehyde in HEPES buffer $(\mathrm{pH} 7.3$ ) and left for $12 \mathrm{~h}$ on a shaker at room temperature. Afterwards, the chips were rinsed two times with DI water and subsequently immersed in an ascending concentration of 2-propanol on a shaker (30\% for $10 \mathrm{~min}$, $50 \%$ for $10 \mathrm{~min}, 70 \%$ for $12 \mathrm{~h}, 90 \%$ for $10 \mathrm{~min}$, three times in $95 \%$ for $5 \mathrm{~min}$, three times in $100 \%$ for $5 \mathrm{~min}$ ). After the last step, the chips were left inside the clean bench for a minimum of $12 \mathrm{~h}$ to dry out. Before SEM imaging the chips were coated with a thin gold layer using a sputter coater (108 auto, Cressington, UK).

\section{Results}

3.1 Micropatterned rGO lines The GO micropatterns were obtained by MIMIC out of an aqueous solution of GO and subsequently reduced with an environmentallyfriendly chemical route using L-aa. The length of the lines was defined by the length of the channels, i.e. by the cutting of the stamp. The width was defined by the structure of the silicon master, which was used to fabricate the PDMS channels for MIMIC. A line width between 2 and $50 \mu \mathrm{m}$ is favorable [17]. In the present study, we mainly focused on lines with $10 \mu \mathrm{m}$ width and a length of about $1 \mathrm{~cm}$. Too large channels would lead to an aggregation of the GO flakes while smaller channels resulted in discontinuous GO patterns. The width of $10 \mu \mathrm{m}$ was chosen because it is comparable to the size of individual cells.

Figure 2A shows a microscopic image of the structured GO patterns on a silicon substrate with $300 \mathrm{~nm}$ silicon dioxide. This substrate was necessary in order to be able to identify the rGO patterns with an optical microscope. Due to the interference effect in DIC microscopy, the color and the contrast of the rGO layer are typically influenced by the wavelength of the illumination and the thickness of the $\mathrm{SiO}_{2}$ layer [21]. The conformal contact formed between the PDMS stamp and the substrate provided separated and continuous patterns. Our patterns were discontinuous when the GO solution did not reach the end of the channels. In general, the MIMIC process is driven by thermodynamical forces. The capillaries are filled by the liquid in order to minimize the free energies of the solid-vapor and solidliquid interfaces. Surface tensions are indicated as $\gamma_{\mathrm{SV}}, \gamma_{\mathrm{SL}}$, and $\gamma_{\mathrm{LV}}$ corresponding to the solid-vapor, solid-liquid and liquid-vapor interfaces. The changes in the Gibbs' interfacial free energy for filling the channels $\Delta G$ with a square cross-section (width $x$ ) from a spherical drop with radius $r$ over distance $\Delta z$ can be expressed as:

$$
\begin{aligned}
\Delta G= & \frac{x^{2} \cdot \Delta z \cdot \gamma_{\mathrm{LV}}}{r} \\
& -\left[3 x \cdot \Delta z \cdot\left(\gamma_{\mathrm{SV}}-\gamma_{\mathrm{SL}}\right)+x \cdot \Delta z \cdot\left(\gamma_{\mathrm{S}^{\prime} \mathrm{V}}-\gamma_{\mathrm{S}^{\prime} \mathrm{L}}\right)\right] .
\end{aligned}
$$

The liquid fills the channel if a positive value for the sum of $\left(\gamma_{S V}-\gamma_{S L}\right)$ and $\left(\gamma_{S^{\prime} V}-\gamma_{S^{\prime} L}\right)$ was obtained. With a negative value the channel does not get filled by the liquid [22]. 


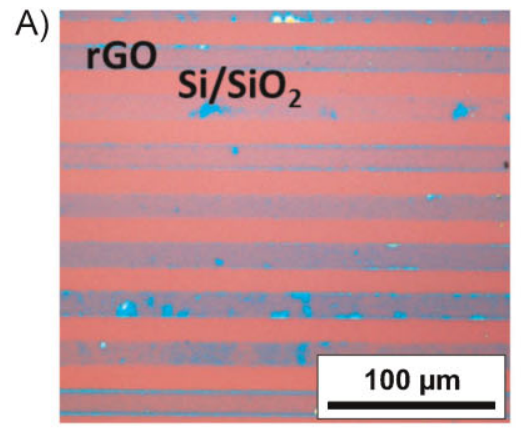

B)

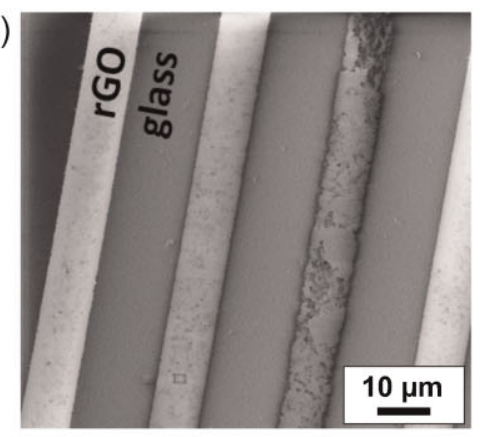

C)
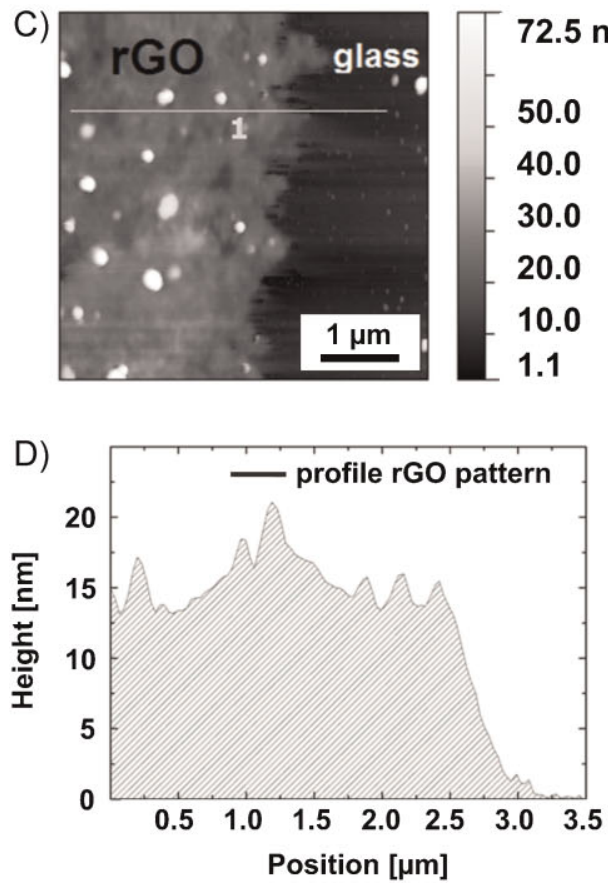

Figure 2 (A) Characterization of rGO patterns (dark lines) by optical microscopy on a $\mathrm{Si} / \mathrm{SiO}_{2}$ substrate. (B) SEM image of patterned rGO on glass. (C) AFM image with a corresponding height profile (D).

In our experiments, most of the GO lines had a homogeneous color, which requires a high density of nonagglomerated flakes with similar flake size. In order to enter the channels, the size of the flakes had to be smaller than $10 \mu \mathrm{m}$. Darker regions of the GO patterns in Fig. 2A are an indication of GO flake agglomeration.
A porous structure of the rGO patterns is visible in the SEM measurement in Fig. 2B. The density of the flakes influences the continuity of the patterns. In Fig. $2 \mathrm{C}$ and D, an AFM image is presented with the corresponding line profile. The profile shows a height of about $15-20 \mathrm{~nm}$ referring to 15-20 rGO single layers [23]. This was the typical thickness of the rGO lines obtained with our fabrication protocol. An rms roughness of 3-4 $\mathrm{nm}$ of the $\mathrm{rGO}$ was obtained from these measurements. The stability of the rGO patterns in aqueous cell culture medium was confirmed by multiple AFM measurements of rGO patterned glass samples stored in M10 medium over 3 days and recording images at same positions each day. No change in morphology or height of the patterns was observed (data not shown).

3.2 Characterization of GO reduction The reduction of the patterned GO lines can be easily assessed optically. A first indicator of a successful reduction process was a visible color change from brown (GO) to black (rGO). Figure 3 shows GO solution in three different stages: GO solution, GO solution reduced for 2 days and GO solution reduced for 4 days, respectively. The reason for the color change is the partial restoration of the $\pi$-network within the carbon structure [23].

Additionally the reduction of GO was monitored by $\mathrm{UV}-\mathrm{Vis}$ spectroscopy. A gradual decrease of the absorbance peak at a wavelength of $280 \mathrm{~nm}$ could be detected over 6 days of reduction (data not shown). The solution reduced for 4 days formed irreversible graphene agglomerates, which has been a key problem in the solution-based chemical reduction of GO as previously described [15].

In this work, chips with the GO line patterns were immersed in L-aa solution, so that the reduction was performed on-chip. With this procedure, we were able to circumvent the agglomeration problem in solution-based reduction. The process improved the electrical conductivity of GO by several orders of magnitude. GO can be considered as an insulator. The reduced form, however, exhibits good electrical conductivity and a fast electron transfer [8].

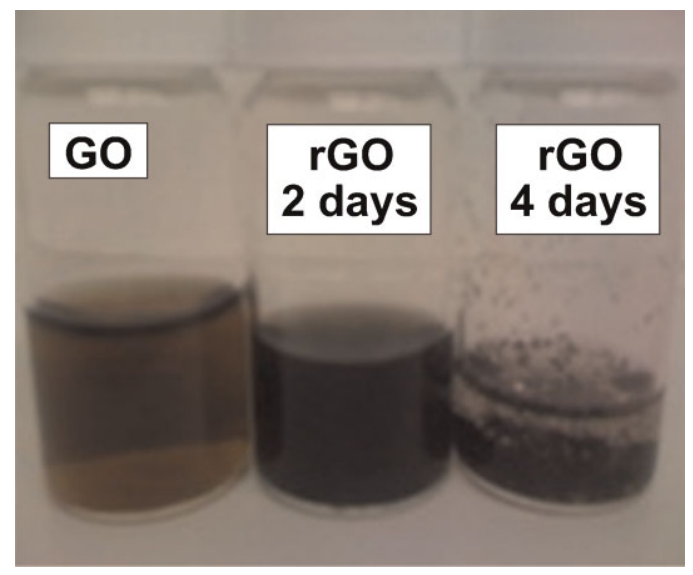

Figure 3 Photograph showing the color change during the reduction of GO in solution by L-aa over 4 days. 
It was previously described that the structure of $\mathrm{rGO}$ contains less defects and oxygen functionalities as compared to GO. During the reduction process, these defects and functionalities are annealed and the excellent electrical conductivity of single layer graphene is partially restored.

Reduction of the patterned GO lines was investigated by IS. Figure 4A shows the IS set-up schematically. During the reduction process, the GO layer changes its initial capacitive characteristics to a more and more resistive behavior. Figure 4B shows a Bode plot $(\log |Z|$ and phase vs. $\log f$ ) of an impedance spectrum before, during and after the reduction of GO.

Starting with non-reduced GO, the electrical behavior was capacitive over the recorded frequency range from $10 \mathrm{~Hz}$ to $1 \mathrm{kHz}$ (Fig. 4B). After 2 days of reduction, the GO had a tendency towards a resistive behavior. This semiresistive behavior is due to the incomplete removal of oxygen in the structure of GO. After 4 days of reduction, the rGO lines can be regarded as perfect resistors.

In our study, the change in the electrical properties can be directly related to the degree of reduction and to the change of color in the aqueous graphene solution. In a first approximation, the impedance spectra in Fig. 4 can be modeled with a simple equivalent circuit composed of a resistance $R$ and a capacitance $C$ in parallel. The change can then be related to a reduced resistance of the rGO lines. However, for electrical recordings from electrogenic cells the obtained reduction was still not strong enough.

A)

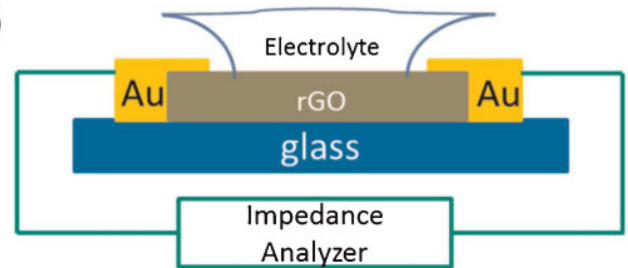

B)
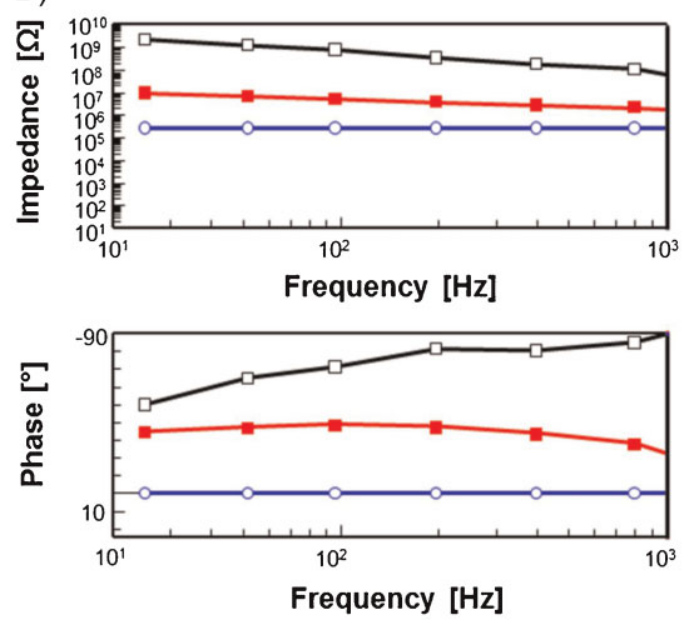

Figure 4 (A) Schematic drawing of the measurement set-up for IS. (B) Impedance spectrum (Bode plot) indicating a gradual impedance change during the reduction of GO over 4 days.
3.3 Characterization of cell growth In first experiments, we compared fully coated substrates of all materials. The surface of the amino-terminated glass with a contact angle of about $56 \pm 1.5^{\circ}$ ( $n=4$ independent measurements) was slightly more hydrophobic than the rGO lines with a contact angle of $51 \pm 2^{\circ}(n=4)$. The HEK 293 cells attached more efficiently to the graphene functionalized glass slides compared to amino-functionalized glass slides and proliferation rate was enhanced (data not shown). This experiment confirmed that rGO promotes cell adhesion and proliferation. Similar results were found by Ruiz et al. [24] with mammalian cell cultures. When we performed the culture experiments on top of the microsized rGO patterns, we observed a strong preference of the cells to adhere on the rGO lines avoiding the amino-modified gaps, which was resulting in large-scale patterns.

Figure 5 shows some exemplary images of HEK 293 cells interfacing rGO patterns after $6 \mathrm{~h}, 1$ day and 2 days in culture. The alignment of the cells on the rGO patterns was already visible after $6 \mathrm{~h}$. The cells strongly preferred the rGO lines during their growth and ongoing proliferation. Even proliferating cells avoided the amino-terminated glass surface in between the lines and tended to grow on top of each other.

We tried to analyze this effect systematically. For this purpose, we recorded microscopy images from three identical patterns at the same position on the glass slides after 1, 2, 3, and 4 days in culture. The results of these experiments are shown in Fig. 6. The cells formed a dense layer on the rGO lines during the first 2 days and the gaps between the rGO lines were clearly visible. During this time, the cells on the line patterns were proliferating and gradually filling the lines. After the second day, the cells started covering the gaps in between the lines as well. However, on day 3 and 4 the number of cells on the lines and inside the gaps was clearly distinguishable with strong statistical significance. After 5 days in culture the cells overgrew the patterns completely forming confluent layers.

The cell adhesion and alignment was verified by SEM (Fig. 7A-C) after 2 days in culture, as well. The nanotopographic roughness of the $\mathrm{rGO}$ lines has a profound influence on cell growth $[4,25]$. Figure $7 \mathrm{~B}$ shows cell growth in multilayers not covering the gap between the lines. Instead of the formation of a confluent layer, a 3D growth was observed with a clear change in morphology from the adhered cell layer compared to the upper layer. Figure $7 \mathrm{C}$ indicates the interface of rGO with HEK 293 cells forming a conformal contact [4]. Cells were attaching directly to the rGO surface and adhered nicely to the substrate. In addition, filaments were growing along the rGO patterns.

4 Discussion It is well-known that cellular alignment is promoted by either chemical or topographic guiding cues. In a previous study using HEK 293 cells, we have already shown that different surface coatings influence the adhesion strength and the average cell-surface distance in the adhesion 

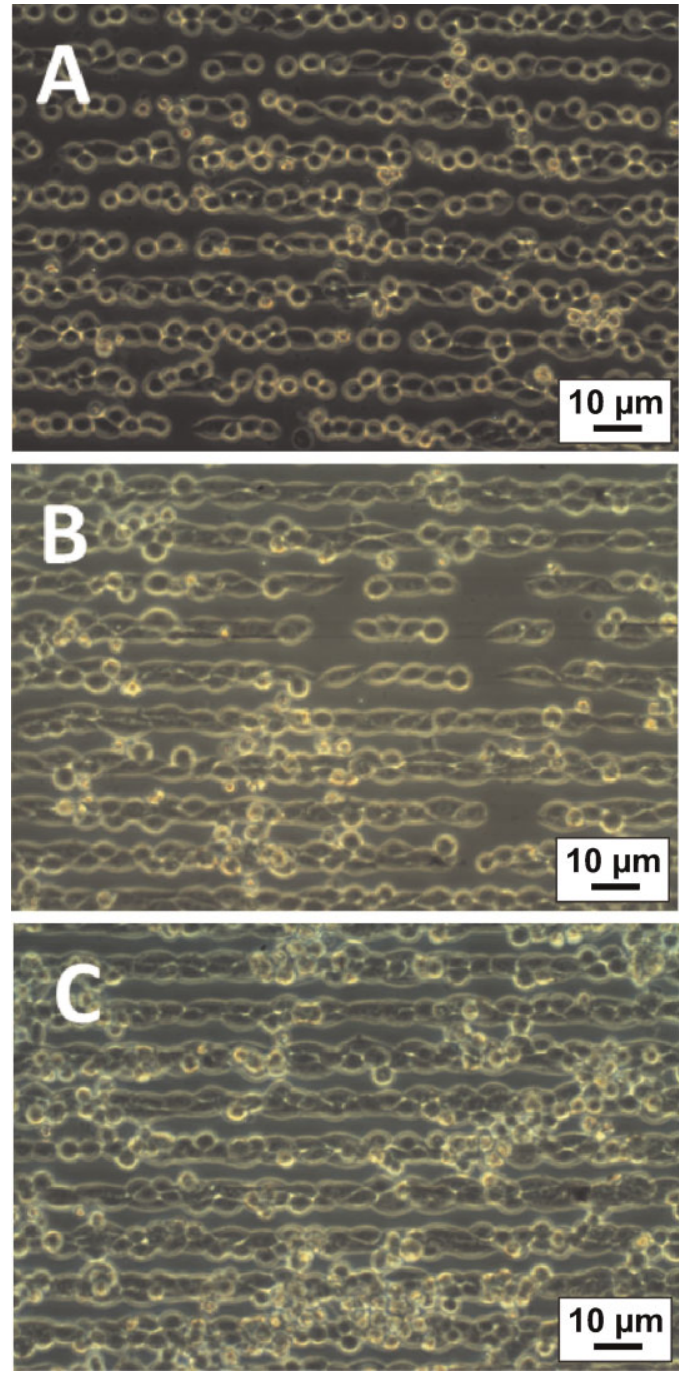

Figure 5 Optical microscopy images showing the growth of HEK 293 cells on rGO patterns after $6 \mathrm{~h}$ in culture (A), after 1 day in culture (B) and after 2 days in culture (C).

region. In these studies, we found that poly-L-lysin was the best coating for this type of cells with the lowest average membrane distance, whereas a coating of fibronectin resulted in the highest amount of focal adhesion contacts [26]. Nevertheless, no coating at all on freshly cleaned glass surfaces was promoting cell adhesion as well. In addition, we have shown that HEK 293 cells typically exhibit three distinct morphologies, namely bowl-shaped, spindleshaped, and a flat morphology [27].

In the current study, all three cell morphologies are distinguishable in the HEK 293 culture as well (Fig. 7). It can clearly be seen that HEK 293 cells adhered preferably to the rGO lines compared to the amino-silanized gaps between lines. This could have two reasons: Either cells are repelled from the amino-silanized glass surface or attracted from the rGO surface. In terms of Gibbs' free energy both surfaces did

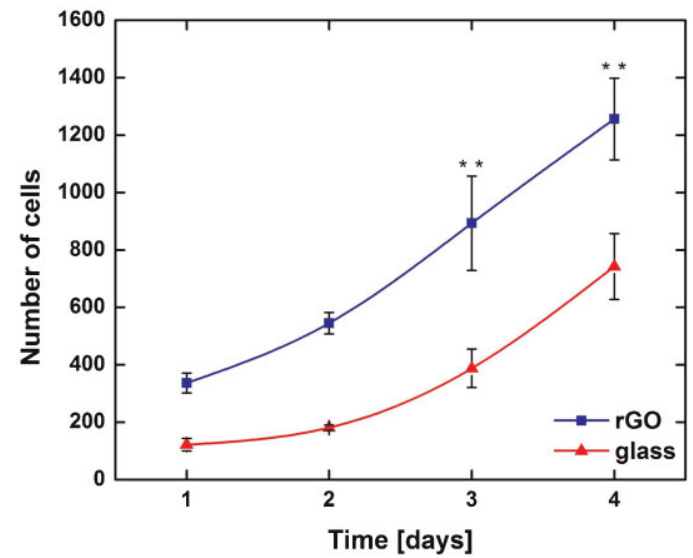

Figure 6 Graph showing the proliferation of cells over 4 days of culture on top of rGO patterns compared to the number or cells growing in between the patterns. There are significantly more cells growing on top of the rGO patterns than on the glass surfaces between the patterns after day $3(n=3,2$-way ANOVA, $\left.{ }^{* *} p<0.01\right)$.

not have a strong difference with $51^{\circ}$ to $56^{\circ}$ water contact angles, respectively. Therefore we assume that the hydrophobicity does not play a big role here. However, as it can be seen in the AFM scans (Fig. 2), the carbon surface exposes a rough surface with possibly a lot of anchoring points for adhesion promoting proteins, which are secreted from the cells themselves. Therefore, we assume that the chemical property and/or the nanotopographic structure of rGO could be the reasons for the patterning effect in our cultures.

5 Conclusions In conclusion, a successful fabrication of GO lines in micrometer width and centimeter length using the MIMIC method was presented. The chemical reduction of the GO line patterns was carried out using an environmentally-friendly method with L-aa improving the conductivity of rGO by at least three orders of magnitude.

When culturing HEK 293 cells on top of these patterns, we found in the first 4 days a strong alignment and close adhesion of the cells onto the rGO lines. Since the cells used in this preliminary study were continuously proliferating, the patterns were overgrown after day 5 in culture. In future studies, we will try to transfer this patterning technique to cell types with lower proliferation rate or to primary cultures such as neurons. The method presented here has several advantages. The procedure for micrometer scale patterning and subsequent reduction is simple, reliable, and scalable to larger areas. The rGO could combine two functions in one: Firstly, it can be used to pattern cell cultures and secondly thanks to the low resistivity in thin layers - it could serve as a transducer material to fabricate future biosensor arrays to address individual electrogenic cells such as neurons in a high lateral resolution. However, in the current stage our protocol could be further improved and standardized. Especially in terms of electronic properties, we need to optimize the sensors to enable such recordings. Compared to 

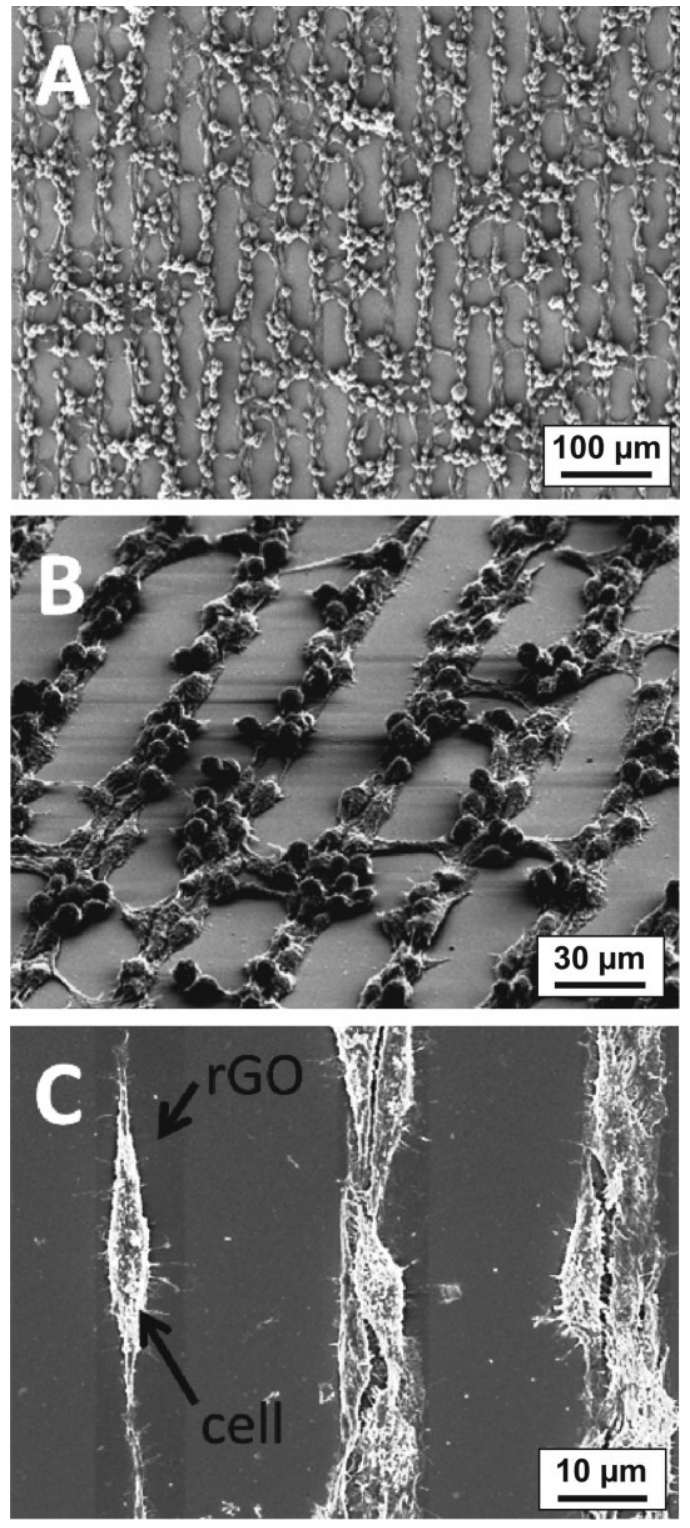

Figure 7 (A-C) SEM images in three different magnifications showing HEK 293 cells aligning on the rGO patterns after 2 days.

the well-established lithography procedures typically used for biosensor fabrication, the presented MIMIC approach is still at a "laboratory level" permitting a reliable mass-production of sensors. At the current stage, it is not clear why the HEK293 cells showed such a strong patterning effect on the structures. This point needs to be clarified in order to transfer our patterning method to other cell types.

As a summary, we established a protocol, which offers the possibility to use graphene as the patterning material for cell guidance and as a transducer material for future fabrication of biosensor devices in parallel. In future work, we will focus in optimizing the electrical properties of our rGO lines and to construct different sensor structures for interfacing electrogenic cells such as neurons.
Acknowledgements The authors would like to thank the technical support of Erik Engelmann and Christian Neu from the mechanical workshop for the measurement cells and shadow masks. In addition Detlev Cassel for clean room support and Rainer Lilischkis for SEM imaging (all University of Applied Sciences Kaiserslautern, Germany) are acknowledged.

\section{References}

[1] M. Kopecek, L. Bacakova, J. Vacik, F. Fendrych, V. Vorlicek, I. Kratochvilova, V. Lisa, E. van Hove, C. Mer, P. Bergonzo, and M. Nesladek, Phys. Status Solidi A 205, 2146-2153 (2008).

[2] M. H. Abouzar, A. Poghossian, A. Razavi, O. A. Williams, N. Bijnens, P. Wagner, and M. J. Schöning, Biosens. Bioelectron. 24, 1298-1304 (2009).

[3] V. Vermeeren, S. Wenmackers, P. Wagner, and L. Michiels, Sensors 9, 5600-5636 (2009).

[4] S. Agarwal, X. Zhou, F. Ye, Q. He, G. C. K. Chen, J. Soo, F. Boey, H. Zhang, and P. Chen, Langmuir 26, 2244-2247 (2010).

[5] C. X. Guo, X. T. Zheng, Z. S. Lu, X. W. Lou, and C. M. Li, Adv. Mater. 22, 5164-5167 (2010).

[6] L. H. Hess, M. Jansen, V. Maybeck, M. V. Hauf, M. Seifert, M. Stutzmann, I. D. Sharp, A. Offenhäusser, and J. A. Garrido, Adv. Mater. 23, 5045-5049 (2011).

[7] D. Lahiri, R. Dua, C. Zhang, I. de Socarraz-Novoa, A. Bhat, S. Ramaswamy, and A. Agarwal, ACS Appl. Mater. Interf. 4, 2234-2241 (2012).

[8] T. Cohen-Karni, Q. Qing, Q. Li, Y. Fang, and C. M. Lieber, Nano Lett. 10, 1098-1102 (2010).

[9] A. Bonanni, A. H. Loo, and M. Pumera, Trends Anal. Chem. (2012).

[10] Y. Dan, Y. Lu, N. J. Kybert, Z. Luo, and A. T. C. Johnson, Nano Lett. 9, 1472-1475 (2009).

[11] X. Dong, Y. Shi, W. Huang, P. Chen, and L.-J. Li, Adv. Mater. 22, 1649-1653 (2010).

[12] P. K. Ang, W. Chen, A. T. S. Wee, and K. P. Loh, J. Am. Chem. Soc. 130, 14392-14393 (2008).

[13] M. J. Fernández-Merino, L. Guardia, J. I. Paredes, S. Villar-Rodil, P. Solís-Fernández, A. Martínez-Alonso, and J. M. D. Tascón, J. Phys. Chem. C 114, 6426-6432 (2010).

[14] S. Stankovich, D. A. Dikin, R. D. Piner, K. A. Kohlhaas, A. Kleinhammes, Y. Jia, Y. Wu, S. T. Nguyen, and R. S. Ruoff, Carbon 45, 1558-1565 (2007).

[15] J. Zhang, H. Yang, G. Shen, P. Cheng, J. Zhang, and S. Guo, Chem. Commun. 46, 1112 (2010).

[16] J. Gao, F. Liu, Y. Liu, N. Ma, Z. Wang, and X. Zhang, Chem. Mater. 22, 2213-2218 (2010).

[17] Q. He, H. G. Sudibya, Z. Yin, S. Wu, H. Li, F. Boey, W. Huang, P. Chen, and H. Zhang, ACS Nano 4, 3201-3208 (2010).

[18] M. Xue, F. Li, J. Zhu, H. Song, M. Zhang, and T. Cao, Adv. Funct. Mater. 22, 1284-1290 (2012).

[19] R. GhoshMoulick, X. T. Vu, S. Gilles, D. Mayer, A. Offenhäusser, and S. Ingebrandt, Phys. Status Solidi A 206, 417-425 (2009).

[20] U. Brunk, V. P. Collins, and E. Arro, J. Microscopy 123, 121-131 (1981).

[21] D. Yoon, H. Moon, Y.-W. Son, J. S. Choi, B. H. Park, Y. H. Cha, Y. D. Kim, and H. Cheong, Phys. Rev. B 2009, 80. 
[22] E. Kim, Y. Xia, and G. M. Whitesides, J. Am. Chem. Soc. 118, 5722-5731 (1996).

[23] G. Eda, G. Fanchini, and M. Chhowalla, Nature Nanotechnol. 3, 270-274 (2008).

[24] O. N. Ruiz, K. A. Shiral Fernando, B. Wang, N. A. Brown, P. G. Luo, N. D. McNamara, M. Vangsness, Y. Sun, and C. E. Bunker, ACS Nano 5, 8100-8107 (2011).
[25] P. Nguyen and V. Berry, J. Phys. Chem. Lett. 3, 1024-1029 (2012).

[26] G. Wrobel, M. Höller, F. Sommerhage, S. Ingebrandt, S. Dieluweit, H.-P. Bochem, and A. Offenhäusser, J. R. Soc. Interf. 5, 213-222 (2008).

[27] F. Sommerhage, R. Helpenstein, A. Rauf, G. Wrobel, A. Offenhäusser, and S. Ingebrandt, Biomaterials 29, 3927-3935 (2008). 